\title{
RNase J1 endonuclease activity as a probe of RNA secondary structure
}

\author{
ROULA DAOU-CHABO and CIARÁN CONDON \\ Institut de Biologie Physico-Chimique, Centre National de la Recherche Scientifique UPR 9073, Université de Paris 7-Denis Diderot, \\ 75005 Paris, France
}

\begin{abstract}
Reliable determination of RNA secondary structure depends on both computer algorithms and experimental probing of nucleotides in single- or double-stranded conformation. Here we describe the exploitation of the endonucleolytic activity of the Bacillus subtilis enzyme RNase J1 as a probe of RNA structure. RNase J1 cleaves in single-stranded regions and, in vitro at least, the enzyme has relatively relaxed nucleotide specificity. We confirmed the feasibility of the approach on an RNA of known structure, B. subtilis tRNA $^{\text {Thr }}$. We then used RNase J1 to solve the secondary structure of the $5^{\prime}$ end of the hbs mRNA. Finally, we showed that RNase J1 can also be used in footprinting experiments by probing the interaction between the $30 \mathrm{~S}$ ribosomal subunit and the Shine-Dalgarno element of the hbs mRNA.
\end{abstract}

Keywords: RNase J1; RNA secondary structure; endoribonuclease; Bacillus subtilis

\section{INTRODUCTION}

Ribonucleic acids (RNAs) can form secondary and tertiary structures that play important roles in regulating transcription, translation, and RNA stability in vivo. RNA structure (more so than sequence) also plays a key role in its recognition by proteins such as regulatory factors, RNA chaperones, or nucleotide modification enzymes. The formulation of testable models in the study of RNA-related processes often depends on reliable predictions of the secondary structure of a given RNA molecule. The consideration of a model of post-transcriptional control at the translational level, for example, might depend on the ability to predict that the ribosome binding site is embedded in a strong secondary structure under certain circumstances. Alternatively, experiments to determine the decay pathway of a particular RNA might be guided by knowing its overall structure. Although mathematical models, including the well-known Zucker MulFold algorithm (http://frontend. bioinfo.rpi.edu/applications/mfold/), are extremely useful and widely exploited to predict RNA structure, many alternative structures are proposed, and determination of the correct structure requires experimental validation.

Reprint requests to: Ciarán Condon, Institut de Biologie PhysicoChimique, Centre National de la Recherche Scientifique UPR 9073, Université de Paris 7-Denis Diderot, 13 rue Pierre et Marie Curie, 75005 Paris, France; e-mail: condon@ibpc.fr; fax: 33-1-58-41-0-20.

Article published online ahead of print. Article and publication date are at http://www.rnajournal.org/cgi/doi/10.1261/rna.1574309.
Many different agents are used to help in the experimental validation of RNA secondary structure. These agents specifically recognize single-stranded (ss) or doublestranded (ds) RNA and modify them by adding chemical groups or by cleaving them at their recognition sites. The treated RNA species can then be analyzed on sequencing gels to identify the site of modification or cleavage at single nucleotide resolution, allowing a determination of which nucleotides are in ss or ds conformation. The experimental data can then be compared with RNA structures predicted by computer modeling to determine the most likely structure. When the theoretical and experimental approaches are combined, they constitute a powerful tool for RNA secondary structure prediction.

RNA modifying or cleavage agents are also useful for the mapping of protein-binding sites on RNA. By comparing the modification/cleavage pattern of an RNA in the presence and absence of its binding protein, one can determine which nucleotides are protected from the action of the agent and therefore constitute the binding site.

The most commonly used RNA cleaving agents are ssspecific ribonucleases (RNases), such as RNase T1, RNase T2, RNase P1, RNase U2, RNase CL3, or RNase A, or the ds-specific RNase V1. These enzymes often have some degree of nucleotide specificity. RNase T1, for example, cleaves $3^{\prime}$ to ss G-residues (Uchida and Egami 1966). RNase CL3 principally cleaves $3^{\prime}$ to C-residues (Boguski et al. 1980), while RNase A cleaves $3^{\prime}$ to C- or U-residues 
(Richards and Wyckof, 1971). RNase T2, however, cleaves broadly $3^{\prime}$ to ss residues with the following hierarchy: A > $\mathrm{G}>\mathrm{C}=\mathrm{U}$ (Irie and Ohgi 2001). RNase P1 also has a broad specificity for single-stranded nucleotides (Oshima et al. 1976). RNase V1 cleaves dsRNAs or stacked ss residues with little nucleotide specificity (Nichols and Yue 2008). Lead (II) is also a popular probe of RNA structure, as it induces cleavage in ssRNA regions. Commonly used RNA modifying agents include $\beta$-ethoxy- $\alpha$-ketobutyraldehyde (kethoxal), 1-cyclohexyl-3'-(2-morpholinoethyl) (CMCT), and dimethyl sulphate (DMS). These chemicals modify the bases of nucleotides not engaged in Watson-Crick (WC) base-pairing interactions and are revealed by primer-extension assay through the ability of these modifications to arrest reverse transcriptase. A powerful new method called selective 2' hydroxyl acylation analyzed by primer extension (SHAPE), which uses N-methylisatoic anhydride (NMIA) and its more reactive derivative 1-methyl-7-nitroisatoic anhydride (1M7), has recently been developed by the Weeks laboratory (Merino et al. 2005; Mortimer and Weeks 2007). These agents modify the $2^{\prime}$ hydroxyl moiety of nucleotides that are not constrained by secondary or tertiary interactions.

During the course of our studies of RNase J1, an essential enzyme of Bacillus subtilis with both endoribonuclease and 5'-to-3' exoribonuclease activity (Even et al. 2005; Mathy et al. 2007), we discovered that this enzyme can be used for the prediction of RNA secondary structure in vitro. Although RNase J1 is thought to have restricted specificity for ss AU-rich sequences in endonuclease mode in vivo (Even et al. 2005; Choonee et al. 2007; Yao et al. 2007; Deikus et al. 2008), the purified enzyme, while maintaining its high degree of specificity for ssRNA, appears to have a more relaxed nucleotide specificity in vitro.

\section{RESULTS}

\section{Use of RNase J1 to confirm a known RNA structure}

We first tested the activity of RNase J1 on an RNA of known secondary and tertiary structure, B. subtilis tRNA ${ }^{\mathrm{Thr}}$. We had previously solved the crystal structure of this tRNA in complex with RNase Z (Li de la Sierra-Gallay et al. 2006). To ensure that we only detected the endonuclease activity of RNase J1, we used a tRNA labeled at its $5^{\prime}$ end with GTP- $\gamma-{ }^{32} \mathrm{P}$, synthesized by T7 RNA polymerase. The 5'-to-3' exoribonuclease activity of RNase J1 is fully in- hibited by a $5^{\prime}$-triphosphate group (Li de la Sierra-Gallay et al. 2008), forcing the enzyme to function solely in endonucleolytic mode. Incubation of 5 '-labeled tRNA ${ }^{\text {Thr }}$ with RNase J1 for different periods of time led to the appearance of a specific pattern of bands (Fig. 1A). Although the reaction shown here has essentially gone to completion after 1 min of incubation with RNase J1, the cleavage pattern is identical at lower concentrations of the enzyme (data not shown). When compared with the known secondary structure of $\mathrm{tRNA}^{\mathrm{Thr}}$ (Fig. 1B), it is clear that RNase J1 cleaves only in ss regions of the tRNA, while nucleotides in base-pairing or stacking interactions are resistant. The anticodon loop is highly accessible to RNase $\mathrm{J} 1$, for example, while all of the anticodon stem, the T-arm, and acceptor stem are resistant. A strong cleavage occurs at nucleotide $\mathrm{U} 48$ of the variable region, which is unconstrained in the three-dimensional structure. RNase J1 cleavages are thus a very good indication of single-stranded or unconstrained RNA conformation.

\section{Use of RNase J1 to solve an unknown RNA structure}

We next applied the technique to an RNA of unknown structure, that of the $5^{\prime}$ end of the $h b s$ mRNA, encoding the $B$.
A

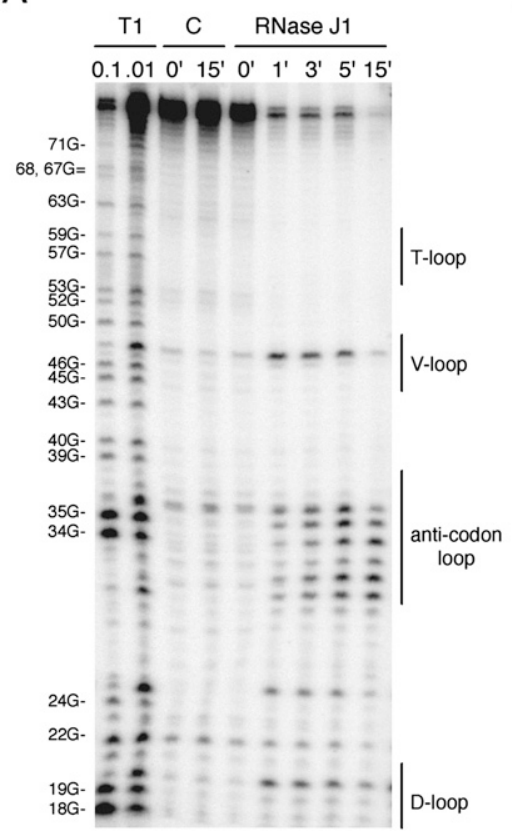

B

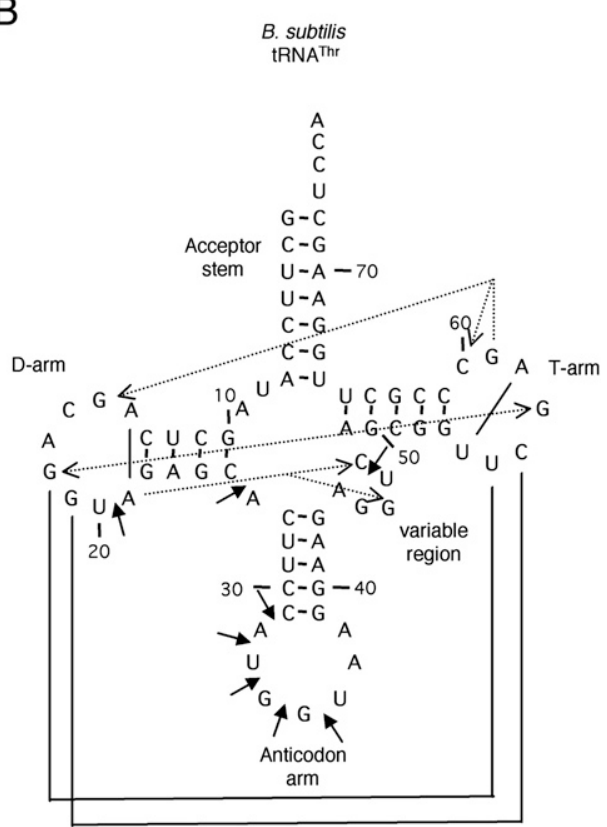

FIGURE 1. Endonucleolytic cleavage of B. subtilis tRNA ${ }^{\text {Thr }}$ by RNase J1. (A) The tRNA was labeled at the $5^{\prime}$ end by GTP- $\gamma-{ }^{32} \mathrm{P}$ and subjected to RNase $\mathrm{J} 1$ digestion for the times indicated. The positions of the G-residues in the tRNA were revealed by RNase T1 cleavage at two different enzyme concentrations and served as a marker. Control reactions incubated for 0 or $15 \mathrm{~min}$ in the absence of enzyme are also shown. The nucleotides corresponding to various features of the tRNA (D-loop, anticodon loop, variable [V] loop, and T-loop) are indicated to the right of the gel. $(B)$ The secondary structure of $B$. subtilis tRNA ${ }^{\text {Thr }}$. The various features of the tRNA (T-arm, D-arm, anticodon arm, and acceptor stem) are indicated. Base-pairing interactions are indicated by solid lines. Stacking interactions are indicated by dotted lines, with the arrowheads indicating the bases involved. RNase J1 cleavages are shown as black arrows. 
subtilis ortholog of the histone-like protein HU. Transcription of the $h b s$ gene begins at either of two promoters, $\mathrm{P} 3$ and $\mathrm{P} 1$, with $\mathrm{P} 3$ yielding transcripts $30 \mathrm{nt}$ longer than P1 (570 versus 540 nucleotides [nt]) (Daou-Chabo et al. 2009). We were interested in solving the structure of these RNAs to understand their high level of stability in B. subtilis in vivo. GTP- $\gamma-{ }^{32} \mathrm{P}$-labeled P3 and P1 transcripts were synthesized by T7 RNA polymerase in vitro and subjected to RNase J1 digestion for short times. The products were run on a sequencing gel alongside an RNase $\mathrm{T} 1$ digestion of the same RNA performed under denaturing conditions to identify the migration positions of the G-residues of the transcript. RNase J1 produces a very reproducible cleavage pattern with both P3 and P1 transcripts (Fig. 2). The intensity of the bands was quite homogenous, except for two hyper-reactive sites at nucleotides $-182 \mathrm{C}$ and $-119 \mathrm{U}$ in the $\mathrm{P} 3$ transcript, suggesting that the purified enzyme has fairly relaxed nucleotide specificity in vitro. Although more of the substrate and longer products disappeared at longer incubation times, the cleavage pattern did not change, suggesting that secondary cleavages were not an issue for these transcripts. The cleavage pattern was compared with alternative structures of the $5^{\prime}$ ends of each $h b s$ transcript proposed by MulFold. The structures that fit best with the data from multiple experiments are shown in Figure 3. The numbering reflects the distance from the first nucleotide of the AUG codon, considered as +1 . The $5^{\prime}$ end of the $\mathrm{P} 1$ transcript is embedded in a stable hairpin $(-16.5 \mathrm{kcal} / \mathrm{mol})$, and this is followed by a second hairpin (hairpin 2, $-14.4 \mathrm{kcal} / \mathrm{mol}$ ) about $20 \mathrm{nt}$ downstream. The 30 extra nucleotides at the $5^{\prime}$ end of the P3
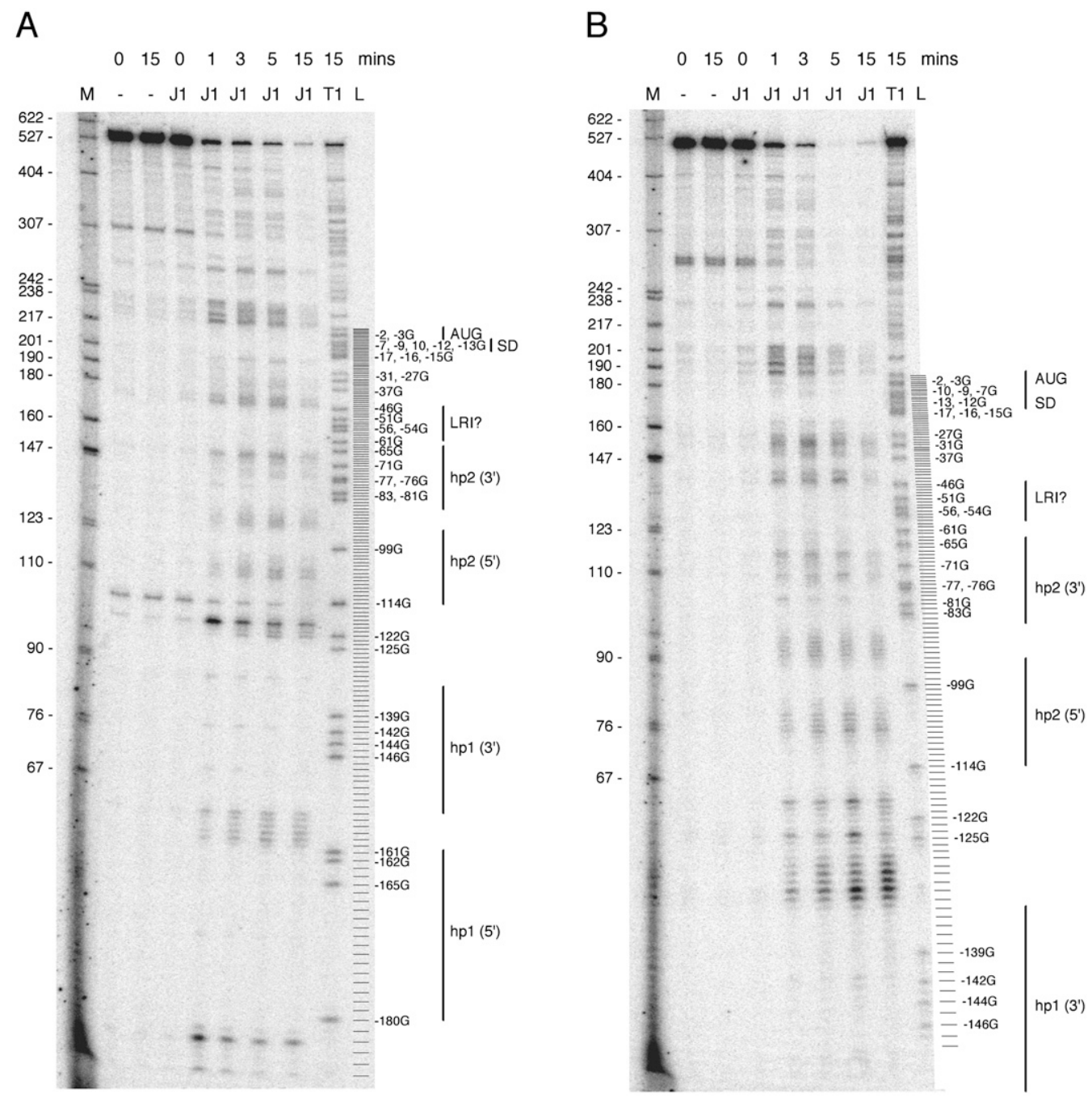

FIGURE 2. Endonucleolytic cleavage of $h b s$ primary transcripts by RNase J1. (A) Cleavage of the P3 transcript. (B) Cleavage of the P1 transcript. Reactions were performed with RNase J1 (J1) or RNase T1 (T1) for the times indicated above the autoradiogram. Control reactions lacking either enzyme are indicated by minus (-) signs. A molecular weight marker is shown in the lane labeled $M$, with the sizes of each band indicated to the left of the autoradiogram. The positions of RNase T1 cleavage relative to the first nucleotide of the hbs initiation codon $(+1 \mathrm{~A})$ are shown to the right of the autoradiogram. A single-nucleotide resolution ladder (L), determined by overexposing the background, is indicated to the right of each gel. Features of the $h b s$ leader region (the $5^{\prime}$ and $3^{\prime}$ strands of hairpins 1 [hp] and 2 [hp2]), the potential long-range interaction (LRI), the Shine-Dalgarno (SD), and AUG sequences are indicated to the right of each gel. 
A

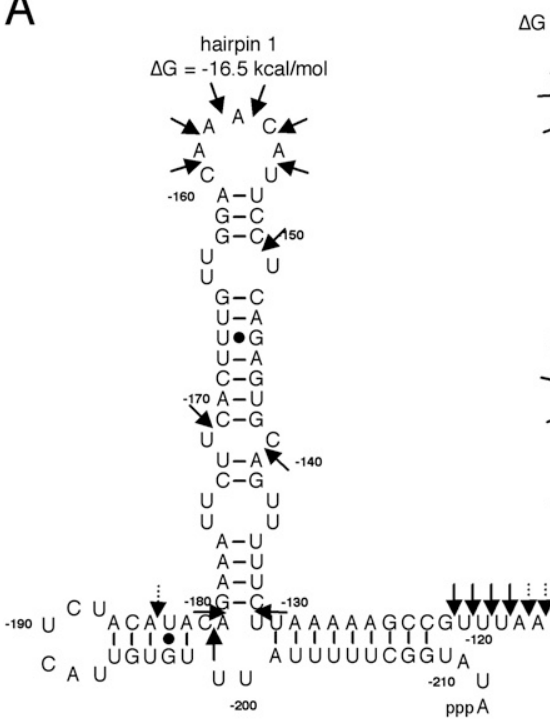

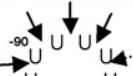

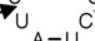

$A-U$
$C-G$
$b-a$

$A-G$
$Y-A$
$C-G$

$C^{U-A} \stackrel{C-B 0}{C-C}$

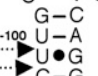

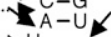

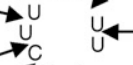

$\mathrm{U}-\mathrm{A}$
$\mathrm{C}-\mathrm{G}$

$U-A-70$

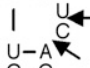

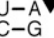

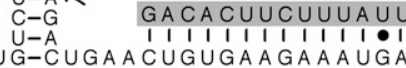

CUGUGA A G A A A U G

P3 transcript

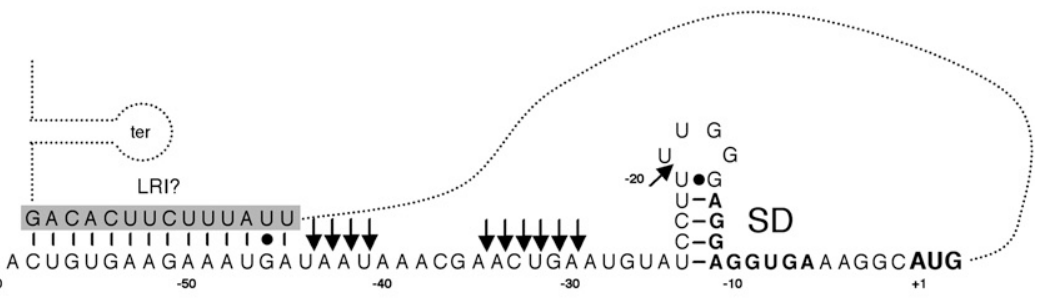

B

hairpin 1 $\Delta \mathrm{G}=-16.5 \mathrm{kcal} / \mathrm{mol}$

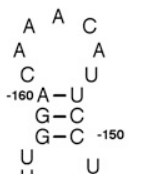<smiles>C1=[Ge]C1</smiles>

$G-C$
$U-A$

$U \bullet G$

C-G

C-G

$U U_{U-A} \mathbb{K}_{-14}$

$C-G$

$U$

$A-U$

$A-U$

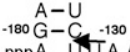

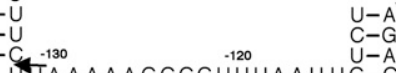

$\Delta \mathrm{G}=-14.4 \mathrm{kcal} / \mathrm{mo}$

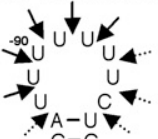

$\underset{C-G}{A-U}$

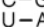

$C-G$

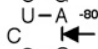
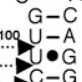

$\stackrel{C-G}{C-U}$

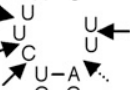

$\mathrm{U}-\mathrm{A}$

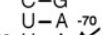

U-A $\simeq$

I

J-AK : LRI?

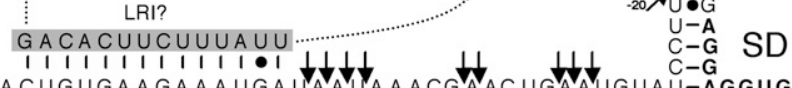

P1 transcript

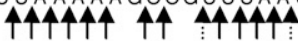

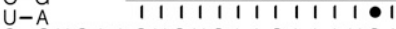

C-G

30

$-10$

\section{C}

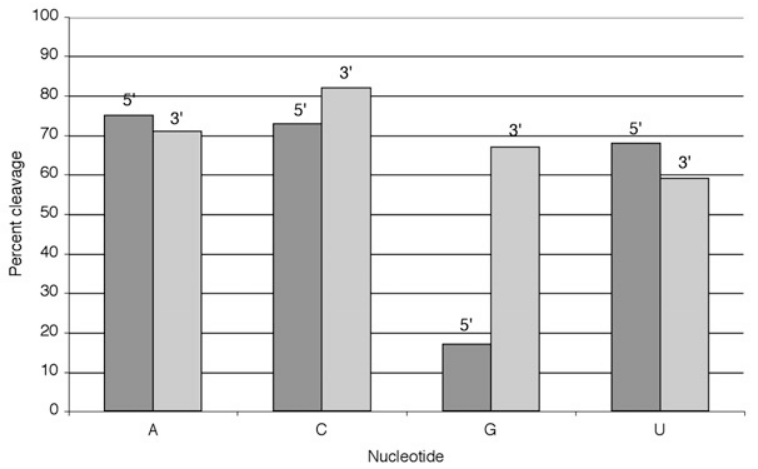

FIGURE 3. Secondary structures of the $5^{\prime}$ ends of the $h b s$ primary transcripts. (A) Secondary structure of the P3 transcript. (B) Secondary structure of the P1 transcript. Nucleotide numbering is relative to the translational start at $+1 \mathrm{~A}$. Positions of RNase J1 cleavage are shown as black arrows. Features of the $h b s$ leader region (hairpins 1 [hp1] and 2 [hp2]), the Shine-Dalgarno (SD), the AUG sequence, and the potential longrange interaction (LRI) are indicated. The intervening nucleotides and transcription terminator (ter) are represented as dotted lines. (C) Cleavage efficiency of RNase J1 5' or 3' to single-stranded A-, C-, G-, and U-residues in the $h b s$ P3 and P1 transcripts. The percentage of cleaved positions was calculated relative to the total number of single-stranded positions for each residue. Although cleavages $5^{\prime}$ to G-residues appear to be underrepresented in this graph, we do not think this is significant (see the text). 
transcript fold back to protect part of the single-stranded region between hairpins 1 and 2 from RNase J1 digestion. As a result, the $5^{\prime}$ end of the $\mathrm{P} 3$ transcript is also embedded in secondary structure and the unavailability of the $5^{\prime}$ end of both primary transcripts likely contributes to the stability of the $h b s$ mRNA.

In both experimentally derived structures, there was a region protected from RNase J1 digestion spanning from the base of hairpin 2 to nucleotide $-45 \mathrm{~A}$, which was predicted to be single stranded in the MulFold model that best fit the experimental data. Only the first $250 \mathrm{nt}$ of each transcript was used to make the original MulFold models. When the full-length transcripts were submitted to the MulFold program, however, we found a 13-nt region, lying just upstream of the transcription terminator, that is fully complementary to the region protected from RNase J1 cleavage. It is possible that a long-range interaction between these sequences is responsible for this protection. It is not clear at this point whether this base-pairing interac- tion predicted from in vitro studies has any significance in vivo, where ribosomes translating the $h b s$ mRNA would be expected to prevent the interaction from occurring.

We used the data from the $h b s \mathrm{P} 3$ and $\mathrm{P} 1$ transcripts to determine the cleavage efficiency of RNase J1 at either the $5^{\prime}$ or $3^{\prime}$ side of each of the four nucleotides (A, C, G, or U) in a predicted single-stranded conformation. Apart from an apparent under-representation of cleavages $5^{\prime}$ to G-residues, RNase J1 showed little nucleotide specificity. Since there are only six predicted unpaired G-residues in the analyzed portion of these two transcripts, is not clear that this statistically significant. Indeed, RNase J1 cleaved 5' to the two G-residues in the anticodon loop of tRNA ${ }^{\text {Thr }}$ quite efficiently (Fig. 1).

\section{Comparison of RNase J1 with a commonly used probe of RNA structure, RNase T2}

To compare the performance of RNase J1 with another commonly used enzymatic probe of RNA secondary struc-
A

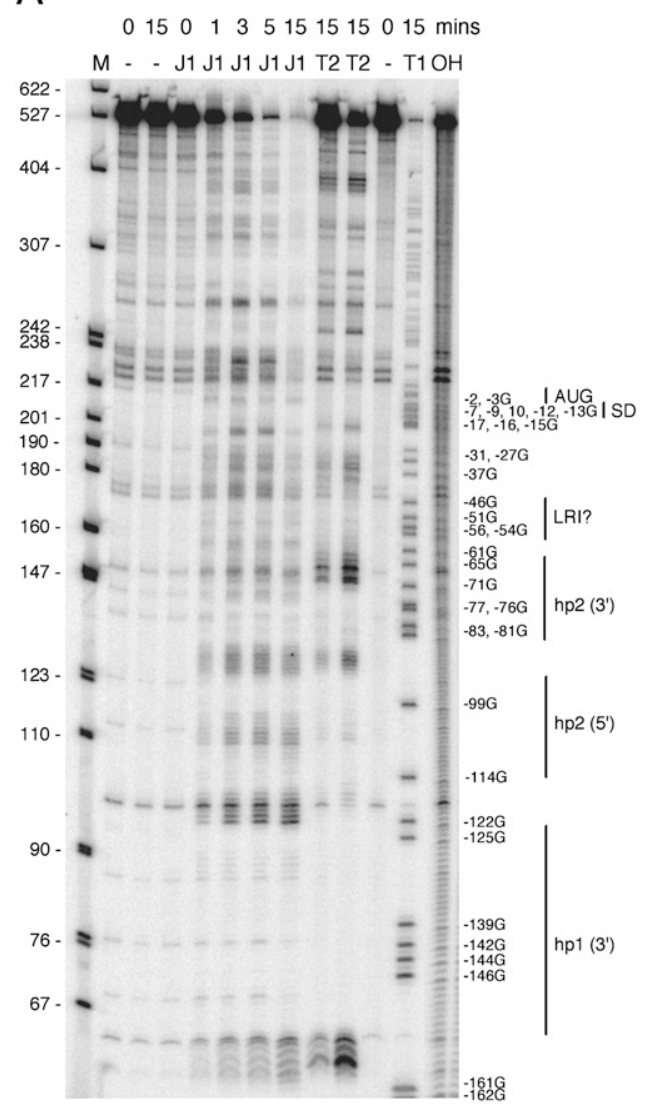

B

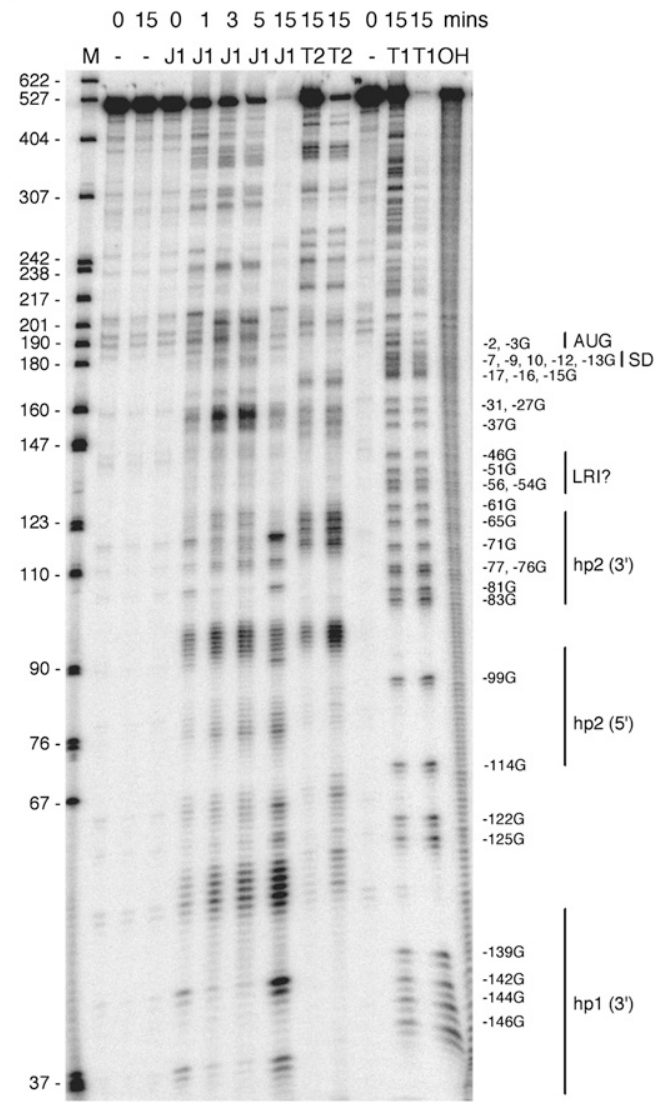

FIGURE 4. Comparison of RNase J1 and RNase T2 reactions. (A) Cleavage of the P3 transcript. (B) Cleavage of the P1 transcript. Reactions were performed with RNase J1 (J1), RNase T2 (T2), or RNase T1 (T1) for the times indicated above the autoradiogram. Two different amounts of RNase T2 were used, $3 \times 10^{-6}$ (lane T2, left) and $3 \times 10^{-5}$ units (lane T2, right). Negative control reactions are indicated by minus (-) signs. A molecular weight marker is shown in lane $M$, with the sizes of each band indicated to the left of the autoradiogram. The positions of RNase T1 cleavage relative to the first nucleotide of the $h b s$ initiation codon $(+1 \mathrm{~A})$ are shown to the right of the autoradiogram. A partial alkaline hydrolysis reaction is indicated by $\mathrm{OH}$. Features of the $h b s$ leader region (the $5^{\prime}$ and $3^{\prime}$ strands of hairpins 1 [hp1] and 2 [hp2]), the potential long-range interaction (LRI), the Shine-Dalgarno (SD), and AUG sequences are indicated to the right of each gel. 
ture, we performed parallel reactions on the $\mathrm{P} 3$ and $\mathrm{P} 1 h b s$ transcripts with RNase J1 and RNase T2. RNase T2 also cleaves nucleotides in a ss conformation, with a preference for A-residues over G-residues, which are both cleaved better than $\mathrm{U}$ or C. RNase J1 and T2 cleaved in similar regions (Fig. 4). The RNase T2 reactions tended to be more constrained, however (apart from a few hyper-reactive sites), presumably because of a more restricted nucleotide preference than RNase J1.

\section{Use of RNase J1 in footprinting assays}

In the course of our experiments on the turnover of the $h b s$ mRNA, we were led to consider the possibility that a strong Shine-Dalgarno (SD) might play an important role in $h b s$ mRNA stability in vivo (Daou-Chabo et al. 2009). The $h b s$ $\mathrm{SD}$ has a potential 12-nt complementarity with the anti-SD sequence at the $3^{\prime}$ end of B. subtilis $16 \mathrm{~S}$ rRNA, and we were interested in probing the interaction between the $30 \mathrm{~S}$ ribosomal subunit and the $h b s$ mRNA. A 147-nt RNA fragment, extending from nucleotide -51 to nucleotide +96 , containing the $h b s$ SD was synthesized in vitro and labeled with a $5^{\prime}$ monophosphate using T4 polynucleotide kinase and ATP $-\gamma^{32}$ P. Although RNase J1 can attack $5^{\prime}$ monophosphorylated RNAs in $5^{\prime}$-to- $3^{\prime}$ exoribonuclease mode, we reasoned that some portion of the RNA would also be cleaved in endonuclease mode. RNAs attacked in exonuclease mode would no longer be detectable once the first labeled nucleotide was removed and only the endonucleolytically cleaved RNAs would be visible on sequencing gels.

The labeled 147-nt RNA was denatured and renatured in the presence of $B$. subtilis 30 S ribosomal subunits and E. coli initiator $\mathrm{tRNA}^{\mathrm{fMet}}$, and the resulting ternary complexes incubated with RNase $\mathrm{J} 1$ for different times. As observed previously, RNase J1 produced a pattern of bands of similar intensity interspaced by protected regions (Fig. 5). In the absence of $30 \mathrm{~S}$ ribosomes and tRNA ${ }^{\mathrm{fMet}}$, RNase J1 cleaved at most positions from -33 to +25 , and this is particularly evident at early times. In the presence $30 \mathrm{~S}$ ribosomes and tRNA $^{\mathrm{fMet}}$, nucleotides -9 to -17 are protected from RNase J1 activity, which corresponds to the predicted SD-antiSD interaction. The RNase J1 protection pattern thus reveals the footprint of the $30 \mathrm{~S}$ ribosomal subunit on the $h b s$ mRNA (Fig. 6). Although 12 nts are potentially complementary to the anti-SD, only 10 of these appear to be tightly base paired. It is not clear why we do not see a greater zone of protection up to and including the AUG start codon, as might be predicted from studies of the $E$. coli ribosome. The stronger reaction in the absence the $30 \mathrm{~S}$ subunit (Fig. 5) suggests that RNase J1 is titrated by the $30 \mathrm{~S}$ subunit and may actually be a substrate for RNase J1. The protected regions beyond nucleotide +25 correspond well with two MulFold-predicted secondary structures within the $h b s$ coding sequence. It should also be noted that

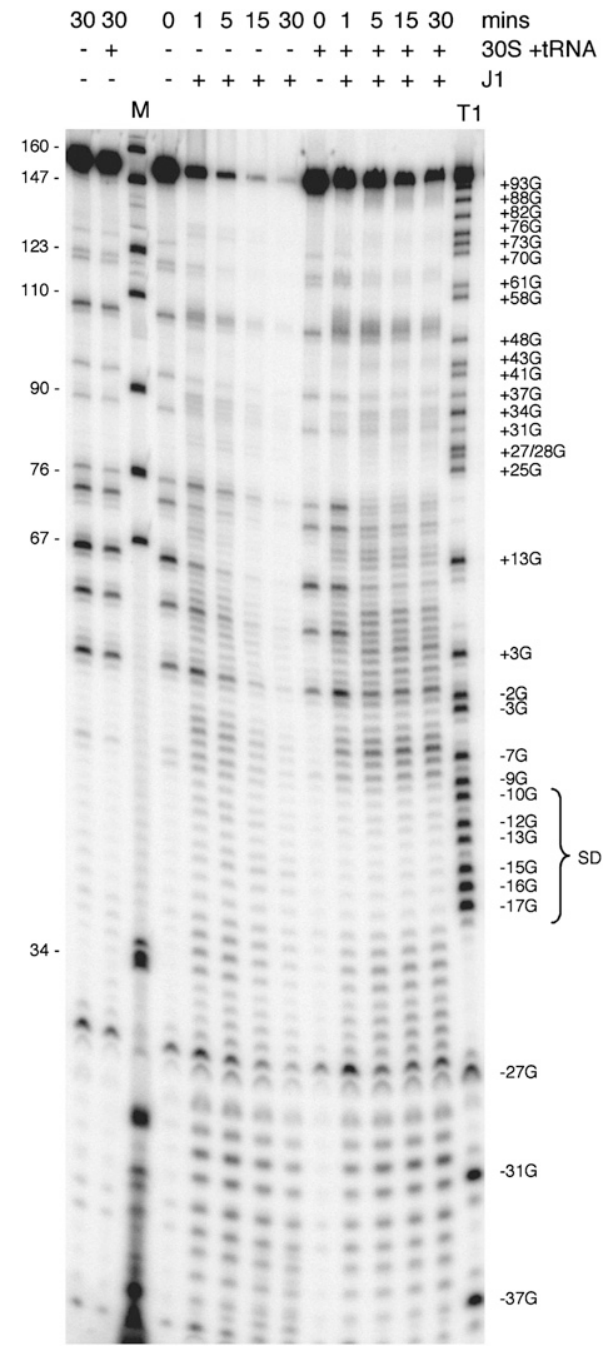

FIGURE 5. Footprint of $30 \mathrm{~S}$ ribosome on $h b s$ Shine-Dalgarno (SD) sequence. RNase J1 digestion of the $h b s$ transcript prebound to $30 \mathrm{~S}$ ribosomal subunits for the times indicated above the autoradiogram. The presence or absence of RNase J1 and 30S subunits is indicated by $(+)$ or $(-)$ signs, respectively. A sequence reaction (lanes $A, C, G, T$ ) and a size standard $(M)$ are used as a size markers. The zone of protection of the SD by $30 \mathrm{~S}$ ribosomes is indicated to the right of the autoradiogram, as are some nucleotides of the $h b s$ transcript referred to in the text.

several cleavages $5^{\prime}$ to G-residues were observed in this experiment, confirming that the apparently poor representation of this particular type of cleavage in Figure 3 was not likely to be significant.

\section{DISCUSSION}

In this work we have exploited the single-strand-specific endonucleolytic activity of RNase J1 to help determine RNA secondary structure. Although the enzyme has a relatively restricted nucleotide specificity in vivo, the purified enzyme has a more relaxed specificity in vitro, cleaving most 


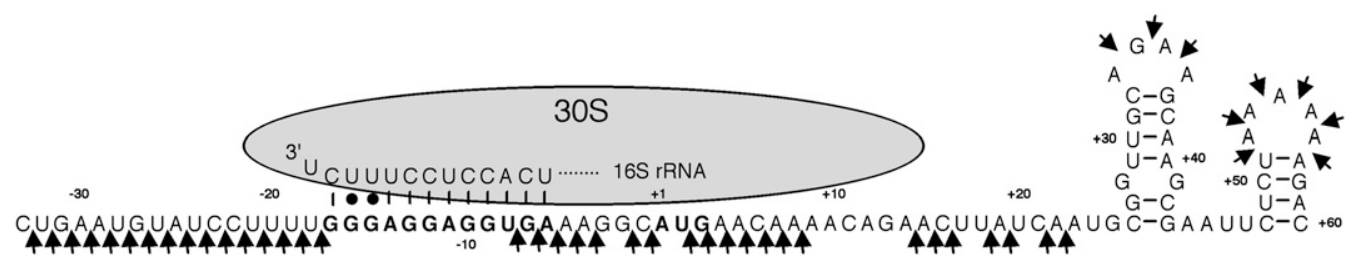

FIGURE 6. Footprint of $30 \mathrm{~S}$ ribosome on $h b s$ Shine-Dalgarno (SD) sequence. Positions of RNase J1 cleavage are shown as black arrows. Nucleotide numbering is relative to the translational start at $+1 \mathrm{~A}$. The $30 \mathrm{~S}$ subunit is shown in gray. The base-pairing interaction between the $3^{\prime}$ end of $16 \mathrm{~S}$ rRNA and the $h b s$ is shown. The SD and AUG sequences are indicated in bold lettering.

nucleotides with apparently similar efficiency. The result is relatively homogenous signals that produce easily interpretable gels. We have confirmed the feasibility of the approach on an RNA of known structure. We also used the method to help us solve the structure of an RNA of interest. Lastly, we showed that we could use the RNase J1 endonuclease activity to demonstrate the footprint of a $30 \mathrm{~S}$ ribosomal subunit through its interaction with the SD of an mRNA.

Direct analysis of RNase J1 cleavages is restricted to relatively short (<300 nt) $5^{\prime}$-labeled RNAs. We have tested different ways of labeling the $5^{\prime}$ end. By using GTP- $\gamma-{ }^{32} \mathrm{P}$ in the in vitro transcription reaction, a $5^{\prime}$ triphosphorylated transcript is generated that is labeled only at the $5^{\prime}$ end. Although the labeling reaction is relatively inefficient due to the dilution of the labeled GTP with cold GTP necessary to complete the transcription reaction, this method has the advantage that all of the visible products of RNase J1 attack are generated by endonucleolytic cleavage. This is because the enzyme's $5^{\prime}$-to- $3^{\prime}$ exonuclease activity is inhibited by $5^{\prime}$ triphosphate. While labeling with polynucleotide kinase and ATP $-\gamma_{-}{ }^{32} \mathrm{P}$ is more efficient than the GTP- $\boldsymbol{\gamma}_{-}{ }^{32} \mathrm{P}$-based method, the presence of a monophosphate group at the $5^{\prime}$ end of the RNA means that a significant proportion of the signal is lost to the $5^{\prime}$-to- $3^{\prime}$ exonuclease activity. Nonetheless, all of the visible products are also the result of endonucleolytic cleavage. The end result is that the two labeling methods are fairly equivalent in terms of their practicality and the results observed.

RNase J1 has both some advantages and some limitations compared with currently available techniques. The ability to directly load the cleavage reactions on gels for short (up to $300 \mathrm{nt}$ ) 5'-labeled RNAs is a considerable advantage over methods requiring primer extension reactions. RNase J1 also appears to cleave all four nucleotides with a high degree of efficiency and performs better than the commonly used broad-specificity enzyme RNase T2 in side-byside assay. The main limitation of the method is that it cannot be used for very long RNAs. Primer extension cannot be used to assay RNase J1 cleavage of longer RNAs, because of the enzyme's $5^{\prime}$-to- $3^{\prime}$ exoribonuclease activity. The downstream fragment of an endonucleolytic cleavage reaction can become a substrate for the enzyme's exonuclease function; thus, bands detected by primer extension assay are not necessarily sites of primary cleavage. Second, relatively large quantities of enzyme are required for reactions, although this is offset by the fact that a C-terminal His-tagged overproducing clone is available, which allows purification of large quantities of the enzyme in a single step. Finally, RNase J1 functions optimally in the presence of magnesium; thus, it may not prove to be quite as effective as RNase T2 under semidenaturing conditions, i.e., in the absence of magnesium.

Although purified RNase J1 appears to have relatively relaxed nucleotide specificity in vitro, like $E$. coli RNase E, it shows a preference for AU-rich single-stranded regions in vivo (Even et al. 2005; Choonee et al. 2007; Yao et al. 2007; Deikus et al. 2008). Indeed, RNase E and RNase J1 can cleave at the same site in the leader region of the thrS gene, encoding threonyl-tRNA synthetase (Condon et al. 1997). It is not clear at this point what leads to the relaxed specificity in vitro; it is possible that the enzyme interacts with other protein partners in vivo that influence its nucleotide preference. Here, we show that as an isolated enzyme in vitro it provides a powerful new option for the experimental evaluation of RNA secondary structure models predicted by mathematical algorithms.

TABLE 1. Oligonucleotides used in this study

\begin{tabular}{ll}
\hline CC424 & ATTAATACGACTCACTATAGCTTCCATAGCTCAGCAGGTAG \\
CC429 & GTAAGCTAGATGCATACACGATCTATATTCAC \\
CC464 & GCTCTAATACGACTCACTATAGGAAATTCTTCACTTTGTTGGACAAAC \\
CC470 & GCTCTAATACGACTCACTATAGGGATATGGCTTTTTATATG \\
CC479 & GCTCTAATACGACTCACTATAGGGAAATGATAATAAACGAACTGAATGTATCC \\
CC570 & GATCGTATCAAAAACAGAGTCAACTGC \\
HP560 & TGGAGCTTCCAAGCGGGCTCGA \\
\hline
\end{tabular}

Nonhybridizing T7 promoter sequences are in italics. 


\section{MATERIALS AND METHODS}

\section{Template preparation}

The templates for the in vitro transcription reaction described below were synthesized by PCR using oligo pairs HP560/CC424 for tRNA ${ }^{\text {Thr }}$, CC470/CC429 for the hbs P3 transcript, CC464/ CC429 for the $h b s$ P1 transcript, and CC479/CC570 for the $h b s$ 147-nt transcript. The sequences of the oligonucleotides are given in Table 1.

\section{GTP- $\boldsymbol{\gamma}-{ }^{32} \mathrm{P}$ labeling of RNAs}

T7 RNA polymerase (Promega or Ambion) was used to synthesize RNAs according to the manufacturer's instructions using UTP, CTP, ATP, and GTP $-\gamma-{ }^{32} \mathrm{P}$ for the first $10 \mathrm{~min}$ of incubation at $37^{\circ} \mathrm{C}$. The purpose of this step is to allow as much incorporation of GTP- $\gamma-{ }^{32} \mathrm{P}$ into the first position of initiated transcripts as possible. Cold GTP is then added and incubation continued for 60 to $90 \mathrm{~min}$ to complete the synthesis of full-length transcripts. The DNA template is degraded by incubation for 15 min with RNasefree DNase (Promega or Ambion). Unincorporated nucleotides are removed using G50 spin columns (GE Healthcare).

\section{Polynucleotide kinase labeling of RNAs}

Unlabeled RNAs are synthesized using T7 RNA polymerase (Promega or Ambion) according to the manufacturer's instructions, except that an eightfold excess of guanosine over the other NTPs is added to the in vitro transcription reaction. This results in about $90 \%$ of the transcripts bearing $5^{\prime}-\mathrm{OH}$ groups. The DNA template is degraded by incubation for $15 \mathrm{~min}$ with RNase-free DNase (Promega or Ambion) and unincorporated nucleotides are removed using G50 spin columns (GE Healthcare). The $5^{\prime} \mathrm{OH}$ bearing RNAs are labeled using polynucleotide kinase (Biolabs) and ATP $-\gamma-{ }^{32} \mathrm{P}$ according to the manufacturer's instructions. Unincorporated nucleotides are once again removed using G50 spin columns.

\section{RNase J1 purification and reaction conditions}

C-terminal His-tagged B. subtilis RNase J1 is purified from E. coli BL21 CodonPlus cells on Cobalt-Talon resin (Clontech) as described previously (Condon et al. 2008). A total of $1 \mu \mathrm{g}$ of RNase J1 is typically incubated for different times with $0.1-0.25$ $\mu \mathrm{g}$ of $5^{\prime}$-labeled RNA in a $5 \mu \mathrm{L}$ reaction volume containing 20 $\mathrm{mM}$ Tris- $\mathrm{HCl}$ ( $\mathrm{pH} 8.0$ ), $8 \mathrm{mM} \mathrm{MgCl}_{2}, 100 \mathrm{mM} \mathrm{NH}_{4} \mathrm{Cl}, 0.1 \mathrm{mM}$ DTT. Reactions are incubated at $37^{\circ} \mathrm{C}$ and are stopped by addition of $5 \mu \mathrm{L}$ of loading buffer (95\% formamide, $20 \mathrm{mM}$ EDTA, $0.05 \%$ bromophenol blue, $0.05 \%$ xylene cyanol).

\section{$\mathrm{RNase} \mathrm{T} 1$ and $\mathrm{T} 2$ reaction conditions}

RNase T1 reactions were performed under denaturing conditions in $20 \mathrm{mM}$ sodium citrate ( $\mathrm{pH}$ 5.0), $1 \mathrm{mM}$ EDTA, $7 \mathrm{M}$ urea buffer containing a total of $1 \mu \mathrm{g}$ of $5^{\prime}$-labeled RNA and yeast competitor RNA. Typically, 0.1 or $0.01 \mathrm{U}$ of RNase T1 (Ambion) were used per $10 \mu \mathrm{L}$ reaction. The samples were heated to $50^{\circ} \mathrm{C}$ to denature the RNA, and then incubated with RNase $\mathrm{T} 1$ at room temperature for $15 \mathrm{~min}$. Reactions were stopped with an equal volume of loading buffer (see above).
RNase T2 reactions were performed in the same buffer conditions as RNase J1 (above) in reactions containing a total of $1 \mu \mathrm{g}$ of $5^{\prime}$-labeled RNA and yeast competitor RNA. Typically, $3 \times 10^{-5}$ or $3 \times 10^{-6} \mathrm{U}$ of RNase T2 (Life Technologies) were used per $10 \mu \mathrm{L}$ reaction. Reactions were incubated at $37^{\circ} \mathrm{C}$ for $15 \mathrm{~min}$ and stopped with an equal volume of loading buffer (see above).

\section{$30 S$ ribosomal subunit footprinting experiments}

5 -labeled 147-nt $h b s$ RNA (0.05 pmol) was denatured in $10 \mathrm{mM}$ Tris-acetate ( $\mathrm{pH} 7.4), 60 \mathrm{mM} \mathrm{NH}_{4} \mathrm{Cl}, 6 \mathrm{mM} \beta$-mercaptoethanol for $4 \mathrm{~min}$ at $80^{\circ} \mathrm{C}$, then frozen in a dry ice/ethanol bath and thawed on ice. Mg-acetate was then added to $10 \mathrm{mM}$. $30 \mathrm{~S}$ ribosomal subunits ( 0.5 pmol per sample in same buffer), isolated from B. subtilis as described (Redko et al. 2008), and $10 \mathrm{pmol}$ E. coli initiator $\mathrm{tRNA}^{\mathrm{fMet}}$ (Sigma) were added and the reactions incubated for $10 \mathrm{~min}$ at $37^{\circ} \mathrm{C}$. RNase J1 (1.8 $\mu \mathrm{g} /$ sample) was added and reactions incubated at $37^{\circ} \mathrm{C}$ for times indicated. Then, $10 \mu \mathrm{L}$ reactions were stopped by addition of $10 \mu \mathrm{L}$ of $95 \%$ formamide, $20 \mathrm{mM}$ EDTA, $0.05 \%$ bromophenol blue, 0.05\% xylene cyanol, and loaded on $8 \%$ polyacrylamide/7 M urea gels.

\section{ACKNOWLEDGMENTS}

We thank L. Bénard, O. Pellegrini, N. Mathy, Y. Redko, and F. Sinturel for helpful discussion. This work was supported by funds from the CNRS (UPR 9073), Université Paris VII-Denis Diderot and the Agence Nationale de la Recherche (ANR).

Received January 23, 2009; accepted April 16, 2009.

\section{REFERENCES}

Boguski MS, Hieter PA, Levy CC. 1980. Identification of a cytidinespecific ribonuclease from chicken liver. J Biol Chem 255: 21602163.

Choonee N, Even S, Zig L, Putzer H. 2007. Ribosomal protein L20 controls expression of the Bacillus subtilis infC operon via a transcription attenuation mechanism. Nucleic Acids Res 35: $1578-1588$.

Condon C, Putzer H, Luo D, Grunberg-Manago M. 1997. Processing of the Bacillus subtilis thrS leader mRNA is RNase E- dependent in Escherichia coli. J Mol Biol 268: 235-242.

Condon C, Pellegrini O, Mathy N, Benard L, Redko Y, Oussenko IA, Deikus G, Bechhofer DH. 2008. Assay of Bacillus subtilis ribonucleases in vitro. Methods Enzymol 447: 277-308.

Daou-Chabo R, Mathy N, Benard L, Condon C. 2009. Ribosomes initiating translation of the $h b s$ mRNA protect it from $5^{\prime}$-to- $3^{\prime}$ exoribonucleolytic degradation by RNase J1. Mol Microbiol 71: 1538-1550.

Deikus G, Condon C, Bechhofer DH. 2008. Role of Bacillus subtilis RNase J1 endonuclease and $5^{\prime}$-exonuclease activities in $\operatorname{trp}$ leader RNA turnover. J Biol Chem 283: 17158-17167.

Even S, Pellegrini O, Zig L, Labas V, Vinh J, Brechemmier-Baey D, Putzer H. 2005. Ribonucleases J1 and J2: Two novel endoribonucleases in B. subtilis with functional homology to E. coli RNase E. Nucleic Acids Res 33: 2141-2152.

Irie M, Ohgi K. 2001. Ribonuclease T2. Methods Enzymol 341: 4255.

Li de la Sierra-Gallay I, Mathy N, Pellegrini O, Condon C. 2006. Structure of the ubiquitous $3^{\prime}$ processing enzyme RNase $\mathrm{Z}$ bound to transfer RNA. Nat Struct Mol Biol 13: 376-377. 
Li de la Sierra-Gallay I, Zig L, Jamalli A, Putzer H. 2008. Structural insights into the dual activity of RNase J. Nat Struct Mol Biol 15: 206-212.

Mathy N, Benard L, Pellegrini O, Daou R, Wen T, Condon C. 2007. $5^{\prime}$-to-3' exoribonuclease activity in bacteria: Role of RNase J1 in rRNA maturation and $5^{\prime}$ stability of mRNA. Cell 129: 681692.

Merino EJ, Wilkinson KA, Coughlan JL, Weeks KM. 2005. RNA structure analysis at single nucleotide resolution by selective $2^{\prime}$-hydroxyl acylation and primer extension (SHAPE). J Am Chem Soc 127: 4223-4231.

Mortimer SA, Weeks KM. 2007. A fast-acting reagent for accurate analysis of RNA secondary and tertiary structure by SHAPE chemistry. J Am Chem Soc 129: 4144-4145.

Nichols NM, Yue D. 2008. Ribonucleases. In Current Protocols in Molecular Biology, Chapter 3, Unit 3.13, Wiley, New York.
Oshima T, Uenishi N, Imahori K. 1976. Simple assay methods for ribonuclease T1, T2, and nuclease P1. Anal Biochem 71: 632634.

Redko Y, Bechhofer DH, Condon C. 2008. Mini-III, an unusual member of the RNase III family of enzymes, catalyses $23 \mathrm{~S}$ ribosomal RNA maturation in B. subtilis. Mol Microbiol 68: 1096-1106.

Richards FM, Wyckoff HW. 1971. Bovine pancreatic ribonuclease. In The enzymes (ed. PD Boyer), pp 647-806. Academic Press, New York.

Uchida T, Egami F. 1966. Procedures in nucleic acids research. I (eds. GL Cantoni, DR Davis), pp 3-13. Harper and Row, New York.

Yao S, Blaustein JB, Bechhofer DH. 2007. Processing of Bacillus subtilis small cytoplasmic RNA: Evidence for an additional endonuclease cleavage site. Nucleic Acids Res 35: 4464-4473. 

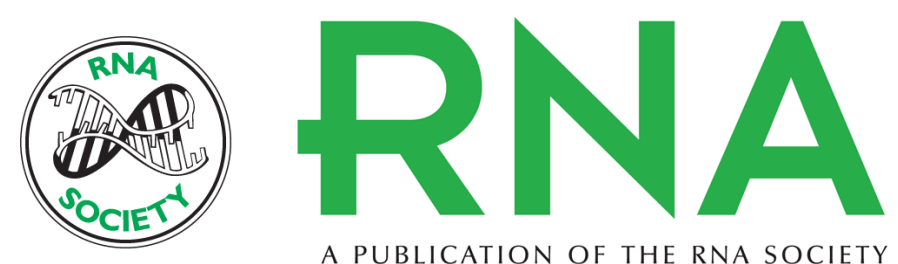

A PUBLICATION OF THE RNA SOCIETY

\section{RNase J1 endonuclease activity as a probe of RNA secondary structure}

Roula Daou-Chabo and Ciarán Condon

RNA 2009 15: 1417-1425 originally published online May 20, 2009

Access the most recent version at doi:10.1261/rna.1574309

\section{References This article cites 16 articles, 2 of which can be accessed free at: http://rnajournal.cshlp.org/content/15/7/1417.full.html\#ref-list-1}

License Email Alerting $\begin{aligned} & \text { Receive free email alerts when new articles cite this article - sign up in the box at the } \\ & \text { Service }\end{aligned}$ top right corner of the article or click here. 